\title{
ВЫСОКОПРОИЗВОДИТЕЛЬНЫЙ АЭРОЗОЛЬНЫЙ ПРОБООТБОРНИК С РЕЦИРКУЛЯЦИЕЙ ЖИДКОЙ ФАЗЫ И ПРЕДВАРИТЕЛЬНЫМ КОНЦЕНТРИРОВАНИЕМ
}

А. Э. Акмалов ${ }^{1}$, Г. Е. Котковский ${ }^{1}$, С. В. Столяров ${ }^{1}$, Б. И. Вердиев², Р. С. Овчинников ${ }^{2}$, А. А. Почтовый², А. П. Ткачук² А. А.Чистяков ${ }^{1}$

1 Национальный исследовательский ядерный университет «МИФИ», Москва

2 Национальный исследовательский центр эпидемиологии и микробиологии имени Н. Ф. Гамалеи, Москва

\begin{abstract}
Обнаружение биогенных аэрозолей является важной задачей при обеспечении безопасности жизнедеятельности человека в современных условиях. На практике часто требуется собирать аэрозоли с больших площадей за малый промежуток времени, что накладывает жесткие ограничения на эффективность пробоотбора, величину прокачиваемого в единицу времени объема воздуха и жизнеспособность собранного биоматериала. В работе представлены результаты по разработке и испытанию устройства отбора аэрозольных проб с высокой объемной скоростью и двухступенчатым концентрированием аэрозольных частиц - виртуального импактора и циклонного коллектора с рециркулирующей жидкой фазой. Приведены алгоритм и результаты расчета параметров импактора, результаты испытаний устройства на модельных сухих и жидких тест-препаратах для частиц размерами 0,5-5 мкм. Подтверждено, что при объемных скоростях пробоотбора выше 4000 л/мин эффективность отбора в жидкую фазу объемом до 10 мл составляет более 20\% массовой доли распыленного аэрозоля, а при объемных скоростях выше 300 л/мин - более 60\% массовой доли. Показано, что устройство сохраняет жизнеспособность отобранного биоматериала. Прооотборник реализован в портативном варианте, обладает возможностью настройки всех параметров отбора и очистки, а также управления по сети.
\end{abstract}

Ключевые слова: аэрозоли, биопатогены, эффективность, пробоотборник, импактор, объемная скорость, циклон

Финансирование: Федеральная целевая программа «Исследования и разработки по приоритетным направлениям развития научно-

технологического комплекса России на 2014-2020 годы», государственный контракт №RFMEFI60117Х0018 с Министерством образования и науки Российской Федерации.

$\searrow$ Для корреспонденции: Геннадий Евгеньевич Котковский

Каширское шоссе, 31, г. Москва, 115409; geko@mail.ru

Статья получена: 27.07.2018 Статья принята к печати: 23.08.2018

DOI: $10.24075 /$ vrgmu.2018.049

\section{HIGH-PERFORMANCE AEROSOL SAMPLER WITH LIQUID PHASE RECIRCULATION AND PRE-CONCENTRATION OF PARTICLES}

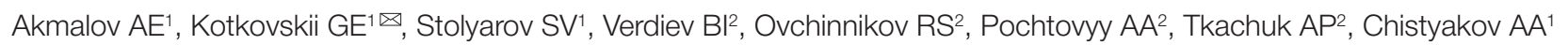

' National Research Nuclear University MEPhl, Moscow

${ }^{2}$ Gamaleya Research Institute of Epidemiology and Microbiology, Moscow

Testing the surrounding environment for the presence of biogenic aerosols is crucial in ensuring its safety for the population. It is often necessary to collect aerosol samples from large areas in short time, which demands excellent particle collection efficiency, a sufficient incoming air flow rate and a capacity to maintain the viability of the collected samples. Below we present the aerosol sampler with a high volumetric flow rate based on a two-stage particle concentration algorithm and consisting of a virtual impactor and a cyclone concentrator with a recirculating liquid phase. We provide all necessary calculations and an algorithm for modeling impactor parameters. The sampler was tested using dry and liquid formulations dispersed into the particles of 0.5 to $5 \mu \mathrm{m}$ in diameter. We demonstrate that at volumetric flow rates over $4,000 \mathrm{l} / \mathrm{min}$ efficiency of particle collection into the liquid phase at a volume of $10 \mathrm{ml}$ makes over $20 \%$ of the total aerosol mass and at volumetric flow rates over $300 \mathrm{l} / \mathrm{min}$ this value is over $60 \%$. The proposed device maintains viability of the collected microorganisms. The sampler is portable, with flexible settings for sampling and cleaning, and can be controlled remotely over the network.

Keywords: aerosols, pathogens, efficiency, sampler, impactor, volumetric flow rate, cyclone

Funding: this work was supported by the Federal Target Program The National System for Chemical and Biological Security of the Russian Federation (20152020), the state contract No. K-27-HИP/148-2 signed by the Ministry of Healthcare of the Russian Federation and the National Research Nuclear University MEPhl.

$\square$ Correspondence should be addressed: Gennadii E. Kotkovskii

Kashirskoe shosse, 31, Moscow, 115409; geko@mail.ru

Received: 27.07.2018 Accepted: 23.08.2018

DOI: $10.24075 /$ brsmu.2018.049

Обнаружение в воздухе патогенных, аллергенных, и иммуногенных микроорганизмов имеет решающее значение при разработке программ, обеспечивающих безопасное существование человека в окружающей среде. С развитием биотехнологий в настоящее время к ранее существующим добавились новые источники биоаэрозольного загрязнения, возникшие в результате применения генной инженерии штаммов микроорганизмов в производстве фармацевтических препаратов, ферментов и пищевых заменителей [1]. Загрязненный воздух может характеризоваться высокими концентрациями микроорганизмов (до $10^{6} \mathrm{KOE} / \mathrm{m}^{3}$ ), являющихся возбудителями инфекционных и аллергических 
респираторных заболеваний человека [2]. Особое внимание требуется уделять целенаправленному загрязнениею воздуха патогенами в результате актов биотерроризма.

Традиционно для обнаружения и количественной оценки биоаэрозолей часто используют методы отбора проб с помощью фильтров, импинжеров или импакторов, в которых микроорганизмы не могут выжить от стресса, вызванного процессом пробоотбора [3]. Между тем, жизнеспособность пробы чрезвычайно важна, например в случае для отбора проб микрофлоры, позволяющих избегать чрезмерного физического воздействия на микроорганизмы, и поддерживающих их физиологические свойства. Наиболее перспективны в этом отношении пробоотборники с жидкой фазой поглощения [4], которые отделяют микроорганизмы от аэрозольных носителей и обеспечивают их точное определение в виде одиночных клеток.

Сформулированы основные требования к устройству пробоотборника для обнаружения биопатогенов в воздушной среде [5-8]. Во-первых, это большая объемная скорость прокачки воздуха, необходимая для обнаружения патогенов низкой концентрации при отборе пробы в разумном временном интервале. Во-вторых, высокая эффективность улавливания аэрозольных частиц и их концентрирование в малом объеме жидкости для дальнейшего анализа. В-третьих, использование мягких, щадящих для микроорганизмов условий осаждения защитных компонент в составе абсорбционной жидкости. Наконец, необходимыми требованиями являются наличие низкого аэродинамического сопротивления у пробоотборника, а также низкий уровень шума и энергопотребление.

Устройства для отбора биогенных аэрозолей из воздушной среды, функционирующие на основе различных физических принципов, разрабатываются и выпускаются на протяжении длительного периода времени. Определенные недостатки присущи каждому типу этих устройств. Создание пробоотборника с высокой эффективностью улавливания патогенов, скоростью отбора пробы более 3000 л/мин, возможностью получения жизнеспособного концентрированного из воздушной пробы материала в малом объеме жидкости, с низким уровнем шума и низким энергопотреблением, до сих пор остается проблемой, требующей своего решения.

Целью работы было создание высокопроизводительного устройства для отбора и концентрирования биоаэрозолей из воздушной фазы для дальнейшего проведения анализа отобранных проб на наличие патогенных бактерий и вирусов.

\section{І. Дизайн эксперимента}

В основу устройства был заложен принцип двухступенчатого последовательного концентрирования, позволяющий работать с большими объемными скоростями прокачки. На первой ступени осуществляется концентрирование частиц за счет принудительного резкого изменения направления движения входного воздушного потока при прокачке через щелевой виртуальный импактор $[9,10]$. Направление выходного воздушного потока, содержащего концентрированные частицы, совпадает с первоначальным направлением входного потока, а его величина в несколько раз меньше. Вторая ступень включает в себя осаждение концентрированных частиц в пленке жидкости, непрерывно циркулирующей в циклонном коллекторе с жидкой фазой $[3,11]$. Увеличение времени циркуляции при продолжающемся поступлении частиц приводит $\mathrm{K}$ возрастанию их концентрации в жидкой фазе. Рассмотрим подробнее основные этапы разработки устройства.

\section{1. Создание виртуального импактора}

При создании виртуального импактора проводили предварительный расчет значений ширины и длины его щелей: входных, куда поступает весь прокачиваемый воздушный поток, и выходных, в которые проходит воздушный поток с уменьшенной объемной скоростью, содержащий концентрированные частицы. Величина входного потока варьировалась от 3000 до 5000 л/мин, размеры селектируемых аэрозольных частиц от 0,5 до 5 мкм. Согласно [12], число Рейнольдса и отношение длины промежутка между входной и выходной щелями к ширине входной щели определяют форму кривой зависимости эффективности селектирования частиц, измеряемой в процентах, от их аэродинамического диаметра. Диаметр, соответствующий 50\%-й эффективности селектирования, определяется как

$$
d_{50}=\left(\sqrt{\frac{9 \eta W}{\rho_{p} C_{C} U}}\right) \times \sqrt{S k_{50}},(1)
$$

где $\rho_{\mathrm{p}}$ - плотность частицы; $C_{\mathrm{C}}$ - поправка Каннингема, учитывающая увеличение подвижности частиц, размер которых сравним со средней длиной пробега молекул газа; U - линейная скорость частицы; $\eta$ - вязкость воздуха или газа; $W$ - ширина щели; $S_{\text {k50 }}$ - число Стокса, соответствующее диаметру $d_{50}$. Расчет состоял в выборе таких размеров щелей, которые при объемных скоростях до 5000 л/мин обеспечивали бы число Рейнольдса

$$
R e=\frac{\rho_{\text {воздуха }} W U}{\eta}
$$

в диапазоне 500-3000 [12]. После этого на основании зависимостей числа $S_{k 50}$ от числа $R e$, полученных в [13], определяли $S_{k 50}$, а далее по формуле (1) вычисляли значение $d_{50}$, которое должно быть не больше минимального требуемого диаметра аэрозолей равного 0,5 мкм. Получили 20 вариантов размеров щелей с различными значениями числа Рейнольдса и $d_{50}$

Следующая стадия разработки виртуального импактора включала в себя построение модели секции виртуального импактора на основании полученных при расчетах параметров щелей, моделирование движения частиц воздуха в секции виртуального импактора и получение распределения частиц воздуха по скоростям в каждой точке области расчета для одной секции. Использовали систему автоматического моделирования Solid Works 2014 с приложением Flow Simulation.

На третьем этапе проводили расчетную проверку эффективности концентрирования частиц при выбранных параметрах с учетом полученного распределения частиц воздуха по скоростям. Использовали специально написанную программу обработки в среде MathLab. Программа рассчитывала смещение массива, включающего в себя 100 аэрозольных частиц, при движении между щелями. Производили пошаговое вычисление координаты каждой частицы с учетом центростремительного ускорения, обусловленного силой Стокса, появляющейся при взаимодействии аэрозольной частицы с воздушным потоком при его повороте в ходе движения между щелями (рис. 1). Оценивали попадание частиц в щель выходного потока. 
На последнем этапе проводили экспериментальную проверку эффективности созданного виртуального импактора при совместной работе с циклонным коллектором.

\section{2. Создание циклонного коллектора}

При создании циклонного коллектора с жидкой фазой использовали расчеты [3], позволяющие определить эффективность захвата частиц жидкой фазой в зависимости от высоты и радиуса цилиндрической трубки, по стенкам которой циркулирует жидкость (рис. 2). Предполагали, что величина входного потока для циклонного коллектора равна величине выходного потока виртуального импактора. Для возможности рециркуляции жидкости в циклонном коллекторе предусматривали отдельный канал.

\section{II. Проведение испытаний пробоотборника}

Изготовленные виртуальный импактор и циклонный коллектор соединяли гибким воздуховодом и испытывали как совместно, так и раздельно, проверяя эффективность сбора аэрозолей. Испытания проводили в ФГБУ 48 ЦНИИ Минобороны России (г. Сергиев Посад), Московском метрополитене и в ФГБУ ФНИЦЭМ им. Н. Ф. Гамалеи Минздрава России (г. Москва).

Испытания в ФГБУ 48 ЦНИИ Минобороны России проводили с использованием модельного сухого тестпрепарата без биопатогенов в течение 10 дней. При проведении испытаний импактор устройства размещали внутри статической аэрозольной камеры, а циклонный коллектор, соединенный воздуховодом с импактором, вне ее. Контроль интегральной концентрации и дисперсного состава аэрозоля, определение его массы в сорбирующих жидкостях проводили флуоресцентным и хемилюминесцентным методами. Эфффективность работы пробоотборных устройств оценивали относительно пробоотборника КПК-3. С помощью импульснопневматического аппарата в статической камере создавали аэрозоль модельного сухого тест-препарата. С помощью прибора КПК-3 и четырехкаскадного импактора Мея

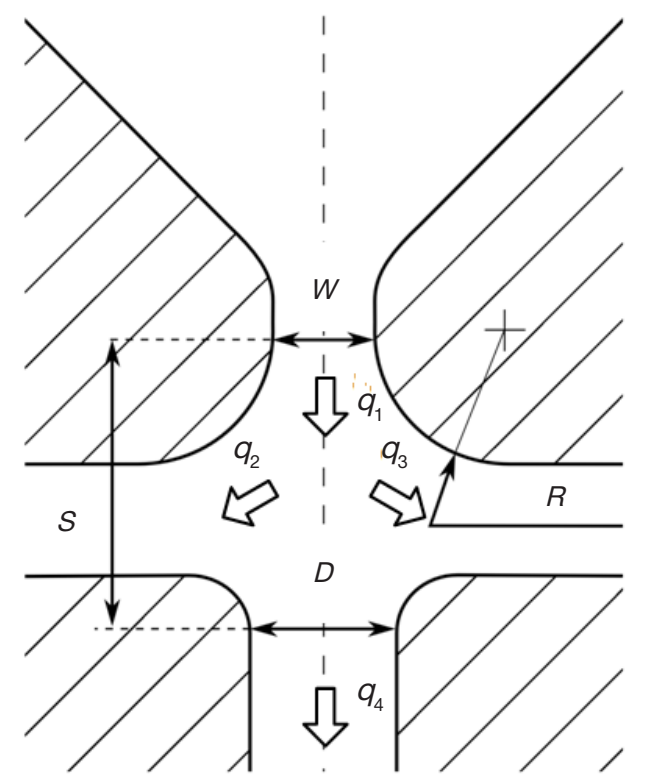

Рис. 1. Схема секции виртуального импактора в попреречном разрезе. $W$ ширина входной щели; D - ширина выходной щели; S - расстояние между щелями; $q_{1}-$ входной поток; $q_{4}$ - выходной поток с концентрированными аэрозольными частицами; $q_{2}$ и $q_{3}$ - отбрасываемый импактором поток оценивали интегральную массовую концентрацию аэрозоля и его дисперсный состав.

Испытания в досмотровых зонах вестибюлей станций «Черкизовская» и «Новокосино» Московского метрополитена проводили для определения эффективности созданного устройства в сравнении с комплектом для забора аэрозольной пробы SASS 4000/2300 (Research International Inc.; США). Собранные образцы направляли для микробиологического анализа и биомолекулярных исследований с целью определения состава микробиоты и ее количественного содержания.

\section{III. Микробиологические исследования полученных образцов}

В работе были использованы готовые агаризованные питательные среды в чашках Петри (ЦФГС; Россия): колумбийский агар с дефибринированной кровью, среда Байрда-Паркера, среда Сабуро с декстрозой и хлорамфениколом, среда Эндо, среда энтерококк-агар и приготовленная самостоятельно среда LB-aгap (lysogeny broth, состав (г/л): 10 г триптона, 5 г дрожжевого экстракта, $5\ulcorner\mathrm{NaCl}, 17$ г агара). Перед посевом питательные среды прогревали до комнатной температуры. При необходимости подсушивали в термостате для удаления конденсата. Для посева образца использовали по 1 чашке каждой питательной среды (по 6 сред на образец).

Посев жидких образцов на питательные среды осуществляли автоматической пипеткой в объеме 0,1 мл на чашку Петри. Жидкость равномерно распределяли по поверхности среды стерильным L-образным шпателем.

Посевы инкубировали при температуре $37^{\circ} \mathrm{C}$ в течение 48 ч. Посевы на среде Сабуро при отсутствии видимого роста продолжали инкубировать при комнатной температуре до 7 сут. включительно.

На каждой из питательных сред проводили учет выросших колоний, описывая их морфологические типы (MT). Каждому МТ присваивали индивидуальный номер и проводили фотографирование колоний. Изолированные колонии отсевали на питательные среды для дальнейшей

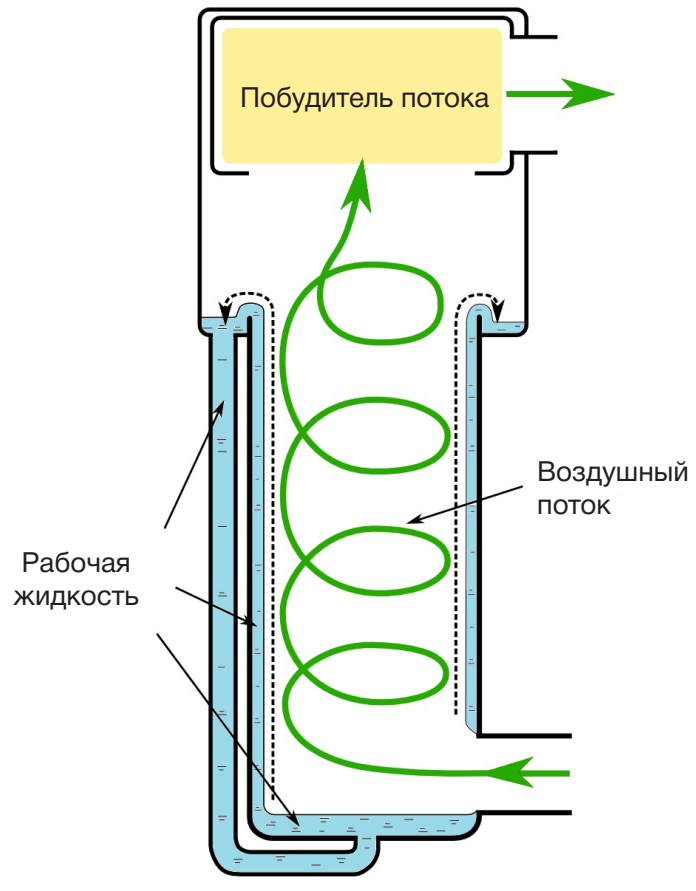

Рис. 2. Схема циклонного коллектора с жидкой фазой 
идентификации и определения чувствительности к антибиотикам. При наличии характерных морфологических признаков осуществляли предварительную идентификацию выросших колоний до уровня рода.

Определение чувствительности выделенных культур к антибиотикам проводили диско-диффузионным методом, с применением тестовых дисков (Himedia; Индия) с антибиотиками: ампициллин, амоксиклав, цефокситин, азитромицин, левофлоксацин, гентамицин, амикацин, тетрациклин, ванкомицин, новобиоцин, бацитрацин, оптохин и стандартизорованной для этих целей среде Мюллера-Хинтона (ООО «ЦФГС», Россия).

Измеряли диаметры зон задержки роста (33Р) культур бактерий вокруг дисков с антибиотиками, затем сравнивали их с референсными значениями и относили культуру к одной из трех категорий: $r-$ устойчива, $s-$ чувствительна, $i$ - умеренно чувствительна.

Испытания в ФГБУ ФНИЦЭМ им. Н. Ф. Гамалеи проводили с целью сравнения эффективности работы созданного устройства и комплекта для забора аэрозольной пробы SASS4000/2300 (Research International Inc.; США) при использовании жидкого модельного тест-препарата. Измерения проводили в боксе микробиологической безопасности специального исполнения «Ламинар-С»
(ЗАО «Ламинарные системы»; Россия). Тест-препарат представлял собой 10\% раствор сахарозы на карбонатном бусрере (рH 9,6) (C3041; Sigma; Германия) с добавлением красителя флуоресцеина натрия в конечной концентрации 1 мкМ. Сравнивали интенсивность люминесценции пробы, собираемой устройствами в течение 5 мин при непрерывном распылении аэрозоля в камере в процессе отбора пробы.

\section{IV. Моделирование параметров виртуального импактора и определение эффективности концентрирования частиц}

В результате проведенных расчетов были получены зависимости эффективности концентрирования аэрозольных частиц с диаметром 0,5-5 мкм от параметров виртуального импактора (рис. 3-5). Эффективность определяли как отношение числа концентрированных, т. е. прошедших в выходной канал (рис. 1), частиц заданного размера к числу частиц во входном потоке.

Эффективность концентрирования возрастает с 18 до 36\% для частиц 0,5 мкм при уменьшении размера входного сопла W от 0,07 до 0,05 см (рис. 3). Однако при переходе к значению $\mathrm{W}$ равному 0,04 см эффеккивность резко падает. Это явление отмечалось в литературе [14] и

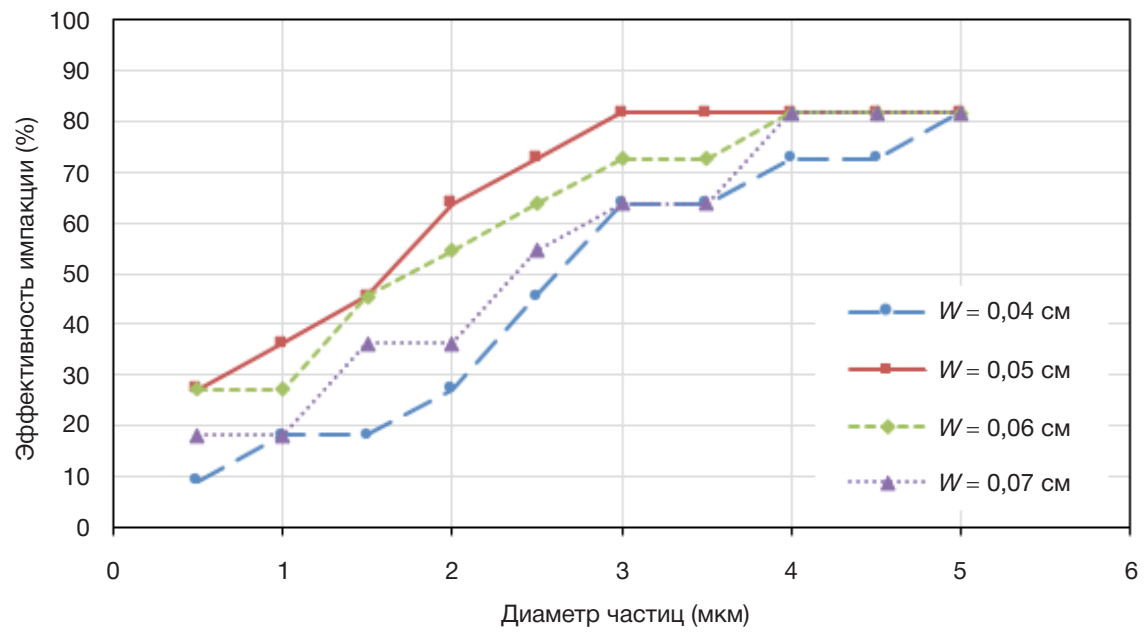

Рис. 3. Зависимость эффективности концентрирования аэрозольных частиц от их диаметра для различных значений ширины входной щели W (рис.1) виртуального импактора. Ширина выходной щели 0,07 см

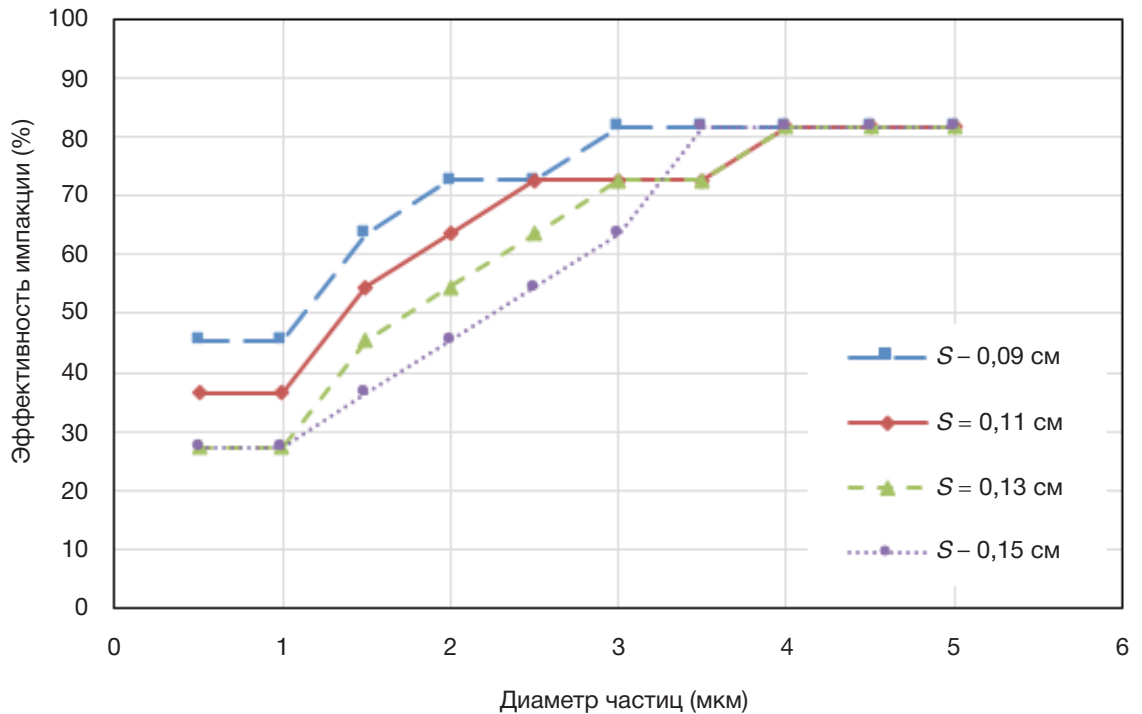

Рис. 4. Зависимость эффективности концентрирования аэрозольных частиц от их диаметра при различных расстояниях между входной и выходной щелью $S$ (рис.1) виртуального импактора. Ширина входной и выходной щели 0,07 см 
указывает на необходимость учета соотношения размеров входной и выходной щелей: входная щель должна быть на 30-40\% меньше выходной для достижения максимальной эффективности концентрирования.

При расстояниях (S) между входной и выходной щелью равных 0,13 и 0,15 см эффективность концентрирования частиц размерами 0,5-1 мкм достигает 27\% (рис. 4). При уменьшении этого расстояния до значения 0,09 см наблюдается увеличение эффективности концентрирования до 45\%. Важно, что для частиц размером больше 4 мкм для всех значений $S$ расчетное значение эффективности не превышает 82\%.

Уменьшение радиуса $(R)$ закругления входной щели до 0,06 см приводит к падению эфффективности концентрирования аэрозольных частиц диаметром 1,5-5 мкм (рис. 5). Увеличение радиуса до 0,12 см поднимает эффективность концентрирования частиц с диаметром 0,5 мкм до 64\%, а частиц с диаметрами от 2,5 мкм до 91\%. Тем не менее к увеличению радиуса закругления входной щели следует относиться с осторожностью, так как расчеты поля скоростей воздушного потока указывают на возможную турбулентность воздушного потока между щелями в этом случае.

Результатами расчета параметров циклонного коллектора с жидкой фазой стали величины, определяющие эффективность импакции (перевода аэрозольных частиц в жидкую составляющую). Это радиус трубки $R=42$ мм, ее высота $H=100$ мм, диаметр входного сопла 15 мм для объемной скорости входного потока $Q=350$ л/мин.

\section{V. Результаты моделирования параметров виртуального импактора и испытания устройства}

На рис. 6 представлена схема устройства отбора аэрозольных проб. Пробоотборник состоит из виртуального импактора, соединенного воздуховодом с циклонным коллектором.

Циклонный коллектор является управляющим модулем для всего устройства. Процесс управления осуществляется при помощи сенсорного экрана и полностью автоматизирован. Отбор пробы устройством протекает в ходе последовательных операций, которые в совокупности составляют законченный цикл. В общем виде цикл состоит из подачи жидкой фазы из резервуара в циклонный коллектор, отбора пробы путем прокачки воздуха через модули устройства, выдачи жидкой фазы для анализа, очистки циклонного коллектора путем промывки. Длительность циклов и значения объемных скоростей прокачки могут регулироваться оператором. Объем жидкой фазы, выдаваемой устройством для дальнейшего анализа, составляет от 2,5 до 10 мл. Устройство может работать в сети по интерфейсу RS-485.

В табл. 1 представлены результаты испытаний устройства в ФГБУ 48 ЦНИИ Минобороны России. Эффективность отбора пробы модельного сухого тестпрепарата созданным устройством отбора аэрозольных проб достигала 20\% от полной массы распыленных частиц. При этом объемная скорость прокачки созданного устройства в 100 раз больше, чем у пробоотборника КПК-3, обеспечивающего 100\% сбора. Циклонный коллектор устройства, функционирующий без виртуального импактора, отбирает до 61\% от полной массы распыленных частиц при скорости прокачки в 6 раз выше, чем у прибора КПК-3.

По результатам испытаний в Московском метрополитене был проведен микробиологический анализ 64 собранных образцов. Выделено и идентифицировано 48 морфологических типов (МТ) микроорганизмов, составляющих микробиоту воздуха и поверхностей метрополитена. Сравнение созданного устройства отбора аэрозольных проб с системой SASS и контрольным методом использования фильтров высокой плотности (нанофильтров) показало, что по количеству морфотипов высеиваемых из собранных образцов они не имеют больших различий (рис. 7).

У 4 из 5 изученных штаммов бактерий, выделенных в метрополитене с использованием клинического протокола, обнаружен тот или иной тип резистентности. У штамма St. haemolyticus MT22 обнаружена множественная резистентность - к макролидам и фторхинолонам. У Streptococcus viridans MT8 множественная резистентность обнаружена к макролидам, аминогликозидам и защищенным ß-лактамам (табл. 2). Это говорит о возможной применимости прибора для контроля бактериологического состава и распространения антибиотикорезистентности в окружающей среде и в клинике.

Результаты количественного определения тотальной ДНК из отобранных проб, проведенного с помощью коммерческого набора PureLink ${ }^{\mathrm{TM}}$ Microbiome DNA Purification Kit (Invitrogen; США) также показали отсутствие

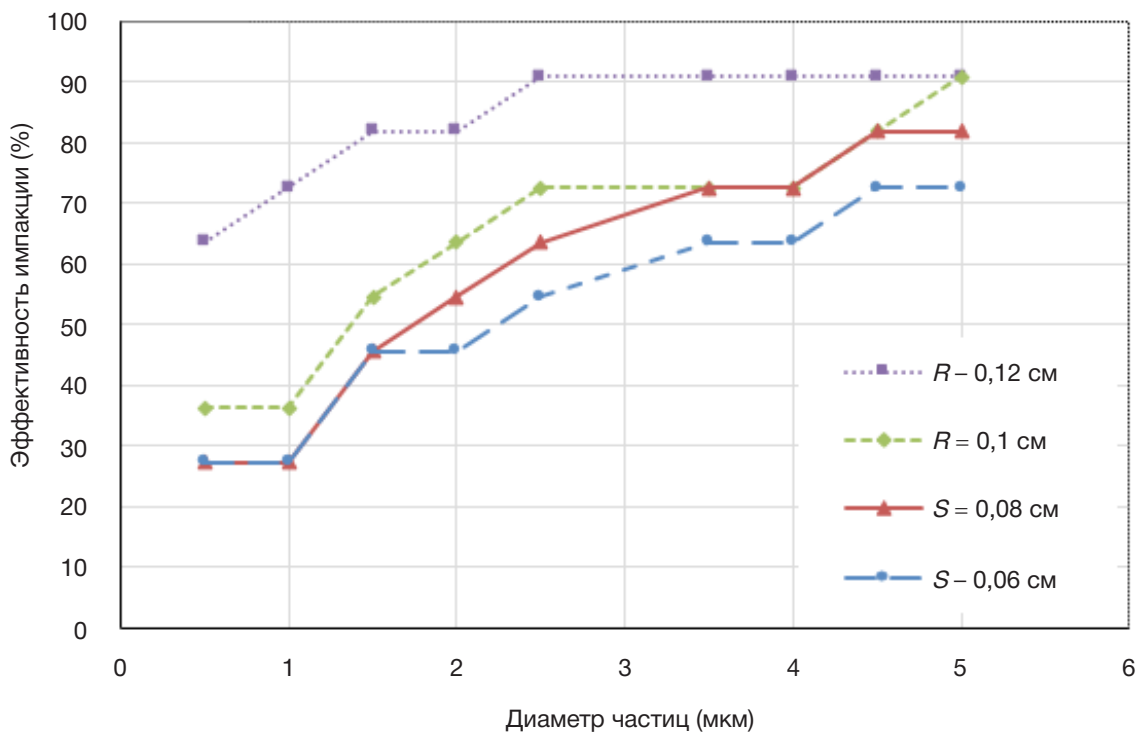

Рис. 5. Зависимость эффективности концентрирования аэрозольных частиц от радиуса закругления $R$ входной щели. Ширина входной и выходной щели $0,07 \mathrm{~cm}$ 
явного преимущества одной из использованных систем пробоотбора. Обе системы на два порядка превосходят контрольный метод с использованием фильтров высокой плотности (нанофильтров).

Испытания в ФГБУ ФНИЦЭМ им. Н. Ф. Гамалеи с жидким тест-препаратом показали, что созданное устройство обеспечивает его сбор на уровне 96\% от системы SASS по результатам 5 измерений с доверительной вероятностью 0,95.

\section{VI. Пути оптимизации работы аэрозольного пробоотборника}

Моделирование параметров виртуального импактора и экспериментальная проверка показывают, что при создании реального устройства основные трудности возникают при обеспечении концентрирования аэрозольных частиц с размерами менее 1 мкм. Получение 50\% эффективности концентрирования таких частиц $\left(d_{50}\right)$ в первую очередь связано с уменьшением ширины входных щелей. Уменьшение ширины щели с 0,5 до 0,4 мм приводит к снижению скорости входного потока в 1,5 раза, что в несколько раз уменьшает эффективность концентрирования. Для поддержания скорости входного потока необходимо во столько же раз увеличить перепад давления, обеспечиваемый вентилятором, что сразу приводит к росту энергопотребления и массогабаритов устройства. Представляется достаточно обоснованным говорить о том, что при требовании концентрирования аэрозольных частиц с размерами менее 1 мкм реально достижимая линейная скорость воздушного потока через входные щели не может быть больше 100 cм/с, а разумная ширина щели не может быть менее 0,5 мкм. В созданном устройстве при работе на максимальной мощности объемная скорость прокачки воздуха не превышает 4500 л/мин.

Эффрективность осаждения аэрозольных частиц в жидкую фазу циклонным коллектором может быть увеличена за счет создания во входном патрубке искусственного распыления мелкодисперсных водяных капель. Отдельной оптимизации требует согласование значений аэродинамического сопротивления выходного тракта виртуального импактора и входного тракта циклонного коллектора, чего в данной работе сделано не было. При дальнейшем совершенствовании устройства

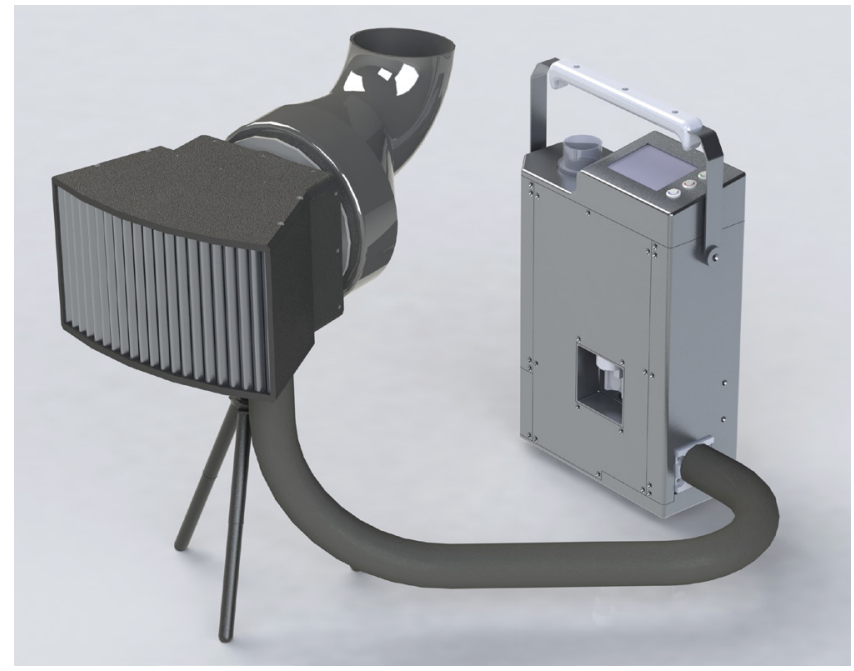

Рис. 6. Устройство Циклон-Био. Изображение устройства в сборе. Слева: импактор. Справа: циклонный коллектор

Таблица 1. Результат испытания прибора с использованием тест-препарата ФГБУ 48 ЦНИИ

\begin{tabular}{|c|c|c|c|c|}
\hline Изделие & $\begin{array}{c}\text { Интегральная массовая } \\
\text { концентрация аэрозоля-имитатора, } \\
\text { мг/л (число измерений } 5, \\
\text { доверительная вероятность 0,95) }\end{array}$ & $\begin{array}{c}\text { Продолжительность } \\
\text { отбора пробы, мин }\end{array}$ & $\begin{array}{l}\text { Объемная скорость } \\
\text { отбора пробы, л/мин }\end{array}$ & $\begin{array}{c}\text { Эффективность отбора } \\
\text { пробы аэрозоля,\% }\end{array}$ \\
\hline \multirow{2}{*}{$\begin{array}{l}\text { Устройство отбора } \\
\text { аэрозольных проб }\end{array}$} & $(1,91 \pm 0,18) \cdot 10^{-3}$ & 2 & 4325 & $16 \pm 1,5$ \\
\hline & $(1,70 \pm 0,16) \cdot 10^{-3}$ & 2 & 4325 & $20 \pm 2,1$ \\
\hline \multirow{2}{*}{$\begin{array}{l}\text { Циклонный коллектор устройства } \\
\text { отбора аэрозольных проб }\end{array}$} & $(3,75 \pm 0,35) \cdot 10^{-3}$ & 2 & 325 & $61 \pm 14$ \\
\hline & $(2,91 \pm 0,27) \cdot 10^{-2}$ & 2 & 325 & $48 \pm 125$ \\
\hline \multirow{2}{*}{$\begin{array}{l}\text { Прибор контроля } \\
\text { концентрации КПК-3 }\end{array}$} & $(3,75 \pm 0,36) \cdot 10^{-3}$ & 2 & 50 & 100,0 \\
\hline & $(2,91 \pm 0,28) \cdot 10^{-2}$ & 2 & 50 & 100,0 \\
\hline
\end{tabular}
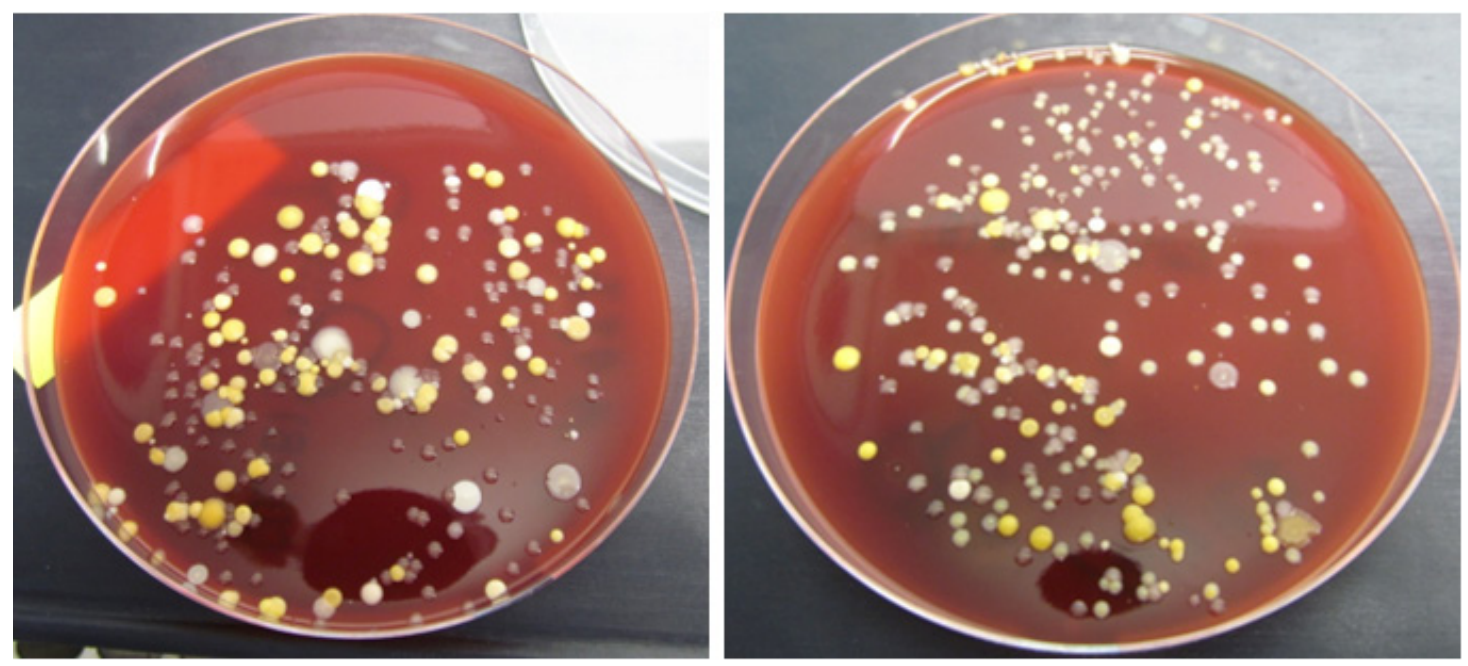

Рис. 7. Суточный посев проб на кровяном агаре, собранных на станции метро «Новокосино» во время утренней пиковой нагрузки. Слева: проба А123, система сбора аэрозоля SASS. Справа: проба А223, система сбора аэрозоля Циклон НИЯУ МИФИ 
Таблица 2. Анализ собранных прибором штаммов на антибиотикорезстентность

\begin{tabular}{|c|c|c|c|c|c|}
\hline Антибиотик & $\begin{array}{c}\text { MT } 11 \\
\text { (Staphylococcus } \\
\text { haemolyticus) }\end{array}$ & $\begin{array}{c}\text { MT } 12 \\
\text { (Staphylococcus } \\
\text { saprophyticus) }\end{array}$ & $\begin{array}{c}\text { MT } 22 \\
\text { (Staphylococcus } \\
\text { haemolyticus) }\end{array}$ & $\begin{array}{c}\text { MT } 24 \\
\text { (Staphylococcus } \\
\text { aureus) }\end{array}$ & $\begin{array}{c}\text { MT } 8 \\
\text { (Streptococcus } \\
\text { viridans?) }\end{array}$ \\
\hline Ампициллин & $\mathrm{R}$ & S & $\mathrm{S}$ & S & 1 \\
\hline Амоксиклав & $\mathrm{H} / \mathrm{O}$ & $\mathrm{H} / \mathrm{O}$ & $\mathrm{H} / \mathrm{O}$ & $\mathrm{H} / \mathrm{O}$ & $\mathrm{R}$ \\
\hline Цефокситин ${ }^{*}$ & S & $S$ & $S$ & S & $\mathrm{H} / \mathrm{O}$ \\
\hline Азитромицин & S & $\mathrm{R}$ & $\mathrm{R}$ & S & $\mathrm{R}$ \\
\hline Левофлоксацин & S & S & $\mathbf{R}$ & S & S \\
\hline Гентамицин & S & S & S & S & $\mathrm{R}$ \\
\hline Амикацин & S & S & S & S & $\mathrm{R}$ \\
\hline Тетрациклин & S & S & S & S & S \\
\hline Ванкомицин & S & S & S & S & S \\
\hline Новобиоцин & S & $\mathrm{R}$ & $S$ & S & $\mathrm{H} / \mathrm{O}$ \\
\hline Бацитрацин & $\mathrm{H} / \mathrm{O}$ & $\mathrm{H} / \mathrm{O}$ & $\mathrm{H} / \mathrm{O}$ & $\mathrm{H} / \mathrm{O}$ & $\mathrm{R}$ \\
\hline Оптохин & $\mathrm{H} / \mathrm{O}$ & $\mathrm{H} / \mathrm{O}$ & $\mathrm{H} / \mathrm{O}$ & $\mathrm{H} / \mathrm{O}$ & $\mathrm{R}$ \\
\hline
\end{tabular}

целесообразным также представляется реализация функции удаленного управления устройством и параметрами отбора по wi-fi сети.

\section{ЗАКЛЮЧЕНИЕ}

В работе проведены расчеты, на основании которых создано устройство для сбора аэрозольных частиц размерами 0,5-5 мкм с высокой объемной скоростью и концентрированием в жидкой фазе. По результатам проведенных испытаний, прибор обеспечивает отбор аэрозолей с объемной скоростью до 4500 л/мин, эффективностью концентрирования до 20\% по массе при объемной скорости более 4000 л/мин и до 61\% по массе при объемной скорости более 300 л/мин. Диапазон размеров селектируемых аэрозольных частиц - 0,5-5 мкм, масса виртуального импактора устройства 7,2 кг, масса циклонного коллектора - 5,6 кг. Электропитание - 220 В переменного тока, 24 и 12 В постоянного тока. Устройство выполнено в пылевлагозащищенном корпусе. По достипнутым показателям эффеективности прибор соответствует лучшим мировым образцам. При этом более 90\% комплектующих деталей прибора - российского производства. Устройство может быть использовано для обеспечения биологической безопасности на транспорте, объектах инфраструктуры, для мониторинга воздушной обстановки на таможенных и пограничных пунктах, экологического наблюдения, а также при оснащении лечебных и научно-исследовательских учреждений Министерства здравоохранения и Министерства обороны Российской Федерации.

\section{Литература}

1. Lacey J, Dutkiewicz J. Bioaerosols and Occupational Lung Desease. J Aerosol Sci. 1994; 25 (8): 1371-1404.

2. Omelianetz G. Biological Hazards as Risks Factors in Microbial Industry. Pharmacol Toxicolog Suppl. 1997; (80): 141-145.

3. Sigaev G, et al. Development of a Cyclone-based Aerosol Sampler with Recirculating Liquid Film: Theory and Experiment. Aerosol Sci and Tech. 2006; 40 (5): 293-308.

4. Henningson EW, Ahlberg MS. Evaluation of Microbiological Aerosol Samplers. A Review. J Aerosol Sci. 1994; (25): 1459-1492.

5. Baron PA, Willeke K. Aerosol Fundamentals. In: Baron PA, Willeke K, editors. Aerosol measurement: principles, techniques and applications. 2nd ed. New York: Wiley-Interscience; 2001. p. $55-60$.

6. McFarlandAR, HYPERLINK"https://www.researchgate.net/profile/ Maria_King4" King MD, HYPERLINK "https://www.researchgate. net/profile/John_Haglund2" Haglund JS, HYPERLINK "https:// www.researchgate.net/scientific-contributions/21610210_ Youngin_Seo" Seo Y. Wetted Wall Cyclones for Bioaerosol Sampling. Aerosol sampling and technology. 2010; 44 (4): 241252. DOI: $10.1080 / 02786820903555552$.

7. Marple V, Olson B, Rubow K. Inertial gtavitational, centrifugal and thermal collection techniques. In: Baron PA, Willeke K, editors. Aerosol measurement: principles, techniques and applications. 2nd ed. New York: Wiley-Interscience; 2001. p. 229-39.
8. Sigaev VI. Novel liquid sampler for aerosols. Abstracts of the 20th annual AAAR Conference; Portland, Oregon. 2001. Abstract 11PG5:406.

9. Solomon PA, HYPERLINK "https://www.researchgate.net/profile/ Matthew_Landis" Landis MS, HYPERLINK "https://www. researchgate.net/scientific-contributions/71734146_Gary_Norris" Norris G, HYPERLINK "https://www.researchgate.net/scientificcontributions/10776481_Michael_P_Tolocka" Tolocka MP. Chemical analyses methods for atmospheric aerosol components. In: Baron PA, Willeke K, editors. Aerosol measurements: principles, techniques and applications. 2nd ed. New York: WileyInterscience; 2001. p. 261-93

10. Saaski E, et al. Concentrator. US Patent 9791353B2. October 2017.

11. Saaski E, et al. Liquid particulate extraction device. US Patent 7846228B1. December 2010.

12. Marple VA, Liu BYH. Characteristics of laminar jet impactors. Envir Sci Technol. 1974; (7): 648-54.

13. Marple $V$. A fundamental study of inertial impactors [dissertation]. Minneapolis (MN): University of Minnesota; 1970.

14. Chen BT, Yeh HC. An improved virtual reactor: design and performance. J Aerosol Sci. 1987; 18 (2): 203-14. 


\section{References}

1. Lacey J, Dutkiewicz J. Bioaerosols and Occupational Lung Desease. J Aerosol Sci. 1994; 25 (8): 1371-1404.

2. Omelianetz G. Biological Hazards as Risks Factors in Microbial Industry. Pharmacol Toxicolog Suppl. 1997; (80): 141-145.

3. Sigaev G, et al. Development of a Cyclone-based Aerosol Sampler with Recirculating Liquid Film: Theory and Experiment. Aerosol Sc and Tech. 2006; 40 (5): 293-308.

4. Henningson EW, Ahlberg MS. Evaluation of Microbiological Aerosol Samplers. A Review. J Aerosol Sci. 1994; (25): 1459-1492.

5. Baron PA, Willeke K. Aerosol Fundamentals. In: Baron PA, Willeke K, editors. Aerosol measurement: principles, techniques and applications. 2nd ed. New York: Wiley-Interscience; 2001. p. 55-60.

6. McFarlandAR, HYPERLINK"https://www.researchgate.net/profile/ Maria_King4" King MD, HYPERLINK "https://www.researchgate. net/profile/John_Haglund2" Haglund JS, HYPERLINK "https:// www.researchgate.net/scientific-contributions/21610210_ Youngjin_Seo" Seo Y.Wetted Wall Cyclones for Bioaerosol Sampling. Aerosol sampling and technology. 2010; 44 (4): $241-$ 252. DOI: $10.1080 / 02786820903555552$.

7. Marple V, Olson B, Rubow K. Inertial gtavitational, centrifugal and thermal collection techniques. In: Baron PA, Willeke K, editors. Aerosol measurement: principles, techniques and applications. 2nd ed. New York: Wiley-Interscience; 2001. p. 229-39.
8. Sigaev VI. Novel liquid sampler for aerosols. Abstracts of the 20th annual AAAR Conference; Portland, Oregon. 2001. Abstract 11PG5:406.

9. Solomon PA, HYPERLINK "https://www.researchgate.net/profile/ Matthew_Landis" Landi MS, HYPERLINK "https://www. researchgate.net/scientific-contributions/71734146_Gary Norris" Norris G, HYPERLINK "https://www.researchgate. net/scientific-contributions/10776481_Michael_P_Tolocka" Tolocka MP. Chemical analyses methods for atmospheric aerosol components. In: Baron PA, Willeke K, editors. Aerosol measurements: principles, techniques and applications. 2nd ed. New York: Wiley-Interscience; 2001. p. 261-93

10. Saaski E, et al. Concentrator. US Patent 9791353B2. October 2017.

11. Saaski E, et al. Liquid particulate extraction device. US Patent 7846228B1. December 2010.

12. Marple VA, Liu BYH. Characteristics of laminar jet impactors. Envir Sci Technol. 1974; (7): 648-54.

13. Marple V. A fundamental study of inertial impactors [dissertation]. Minneapolis (MN): University of Minnesota; 1970

14. Chen BT, Yeh HC. An improved virtual reactor: design and performance. J Aerosol Sci. 1987; 18 (2): 203-14. 